\title{
Capacity Estimation of the Very Short-Range Electromagnetic Underwater Channel Based on Measurements
}

\author{
Jesús López-Fernández, Unai Fernández-Plazaola, and Jose F. Paris \\ Communications Engineering Department, Malaga University, Campus Teatinos, 29071 Malaga, Spain \\ Correspondence should be addressed to Jesús López-Fernández; jlf@ic.uma.es
}

Received 9 May 2014; Revised 11 July 2014; Accepted 13 July 2014; Published 22 July 2014

Academic Editor: Paschalis Sofotasios

Copyright (C) 2014 Jesús López-Fernández et al. This is an open access article distributed under the Creative Commons Attribution License, which permits unrestricted use, distribution, and reproduction in any medium, provided the original work is properly cited.

\begin{abstract}
The significant attenuation experienced by electromagnetic waves in sea water is the main reason why acoustic waves are generally preferred in underwater communication. Nevertheless, acoustic waves have various drawbacks. For example, they are negatively affected by factors such as mechanical noise, slow propagation speed, and, particularly, low bandwidth, which leads to digital links at a lower bit rate. However, in short-range links, these problems can be overcome by reconsidering the use of electric current communications. For instance, data collected by remote-control vehicles in offshore oil and gas and renewable energy plants can be transmitted at distances of even $1 \mathrm{~m}$ or less. This study uses previous frequency response measurements taken in deep water to explore the capacity of a short-range electromagnetic underwater channel. Because of water movement, the nonstatic position of the vehicle when the transmission occurs means that the channel is regarded as randomly time-variant. A statistical model is proposed and the ergodic capacity is calculated for a $7 \mathrm{MHz}$ bandwidth channel at distances ranging from $0.5 \mathrm{~m}$ to $5 \mathrm{~m}$ as well as for different values of transmitter power. The results of this study reflect capacity values of tens of kbps at distances of approximately $5 \mathrm{~m}$ to several Mbps at distances of less than $1.5 \mathrm{~m}$.
\end{abstract}

\section{Introduction}

Nowadays real-time monitoring of marine environment and supervision of undersea equipment health of oil and gas companies are demanding wireless underwater digital links with growing digital speed. Three technologies have been considered for this purpose over the years: acoustic, opti$\mathrm{cal}$, and electromagnetic. Acoustic waves experiment low attenuation in sea water and, hence, long distance links (up to $20 \mathrm{~km}$ ) can be achieved but the allowable bandwidth is very limited (leading to a maximum digital speed of around $40 \mathrm{kbps}$ [1]). A higher bandwidth can be obtained with radio frequency electromagnetic waves at the cost of a much higher attenuation and, hence, shorter links. Optical technologies are being considered due to their extremely high bandwidth. However, the need for line of sight and precise alignment between transmitter and receiver as well as their susceptibility to turbidity and marine fouling imposes a critical constraint on their underwater application. In [2] an up-to-date overview of these technologies is presented.
Underwater wireless links have conventionally been achieved using acoustic waves. This is the unique option for long range links $(>100 \mathrm{~m})$. With the advent of remotely operated vehicles (ROV) data can be collected from sensors at very small distances $(<1 \mathrm{~m})$ (see Figure 1$)$. In this new scenario, the use of electromagnetic waves can be readdressed as it may greatly exceed the $\approx 40 \mathrm{kbps}$ achieved (in the most favorable case) by acoustic means.

An excellent overview of the use of electromagnetic waves for underwater communications from the beginning of the last century to present day is found in [3]. However, the information about data rates attained through an electromagnetic underwater channel is very scarce in the literature.

In [2] data rates over $8 \mathrm{kbps}$ are predicted for ranges under $10 \mathrm{~m}$ using a binary phase shift keying (BPSK) modulation scheme. Experimental measurements carried out both in [1] and later in [4] report data rates up to $1 \mathrm{Mbps}$ at ranges shorter than $1 \mathrm{~m}$. Moreover, Wireless For Subsea (WFS) company [5] delivers a current line of products offering data rates from 100 bps $(<30 \mathrm{~m})$ to $1 \mathrm{Mbps}(<1 \mathrm{~m})$. 


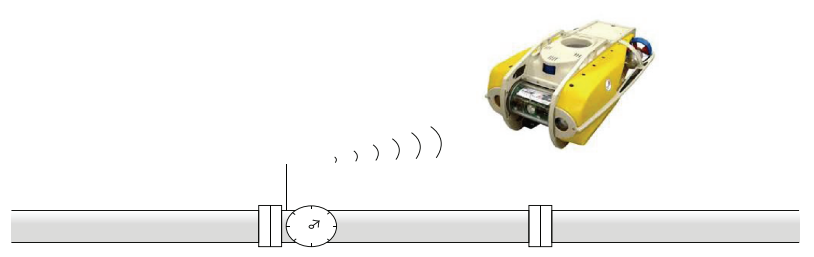

FIGURE 1: Contactless pressure data collection from subsea gas pipe by a remotely operated vehicle.

In this paper the electromagnetic underwater channel capacity is explored for the case of very short-range $(<5 \mathrm{~m})$, deep sea communication. The starting point for this work is [6] where the authors explore the characteristics of this type of channel by conducting a set of experiments focused on obtaining a model for the frequency response.

This paper is organized as follows. In Section 2 a model for frequency response of the channel is described for a separation between transmitter and receiver of $0.1 \mathrm{~m}$. Based on the electric field radiated by a linear dipole antenna the frequency response model is extrapolated to an arbitrary range in Section 3. Section 4 explores the capacity of the channel assuming a statistical model that accounts for the random fluctuation of the receiver due to water movement. Finally, conclusions are extracted in Section 5.

\section{Channel Frequency Response}

In [6] magnitude and phase delay of the underwater channel transfer function were measured employing OFDM probe signals with 128 carriers, total bandwidth $6.25 \mathrm{MHz}$, lower band edge $100 \mathrm{kHz}$, and QPSK modulation. Both transmitter and receiver units were equipped with linear dipole antennas. The experiment in the ocean was carried out at a depth of $5 \mathrm{~m}$ (boundary free) and at $r_{0}=0.1 \mathrm{~m}$ separation between transmitter and receiver.

The collected data were then used to fit different frequency response models corresponding to different approximations of the electromagnetic field propagation in a conducting homogeneous medium.

Three models for the frequency response were considered. The first one corresponds to a far field approximation of the waves in a highly conducting media resulting in an exponential dependence with the square root of the frequency, $\left|H_{r_{0}}(f)\right|=A_{1} e^{-\alpha_{1} \sqrt{f}}$. In the second one the conductivity is assumed to be moderate yielding the model $\left|H_{r_{0}}(f)\right|=A_{2} e^{-\alpha_{2} f}$. In the last two expressions $A_{1}, A_{2}$, $\alpha_{1}$, and $\alpha_{2}$ are the fitting parameters, and $r_{0}$ is the distance between transmitter and receiver. Finally, in the third model the far field approximation is not considered. This yields a rational-polynomial model formulated as

$$
\left|H_{r_{0}}(f)\right|=\frac{p_{1} f^{2}+p_{2} f+p_{3}}{q_{1} f^{2}+q_{2} f+q_{3}} e^{-\alpha f}
$$

where $p_{1}, p_{2}, p_{3}, q_{1}, q_{2}, q_{3}$, and $\alpha$ are the fitting parameters. Best results were obtained with this rational-polynomial
TABLE 1: Frequency response fitting parameters.

\begin{tabular}{lcccccc}
\hline$p_{1}$ & $p_{2}$ & $p_{3}$ & $q_{1}$ & $q_{2}$ & $q_{3}$ & $\alpha$ \\
\hline $3.4 e-5$ & $1.2 e-4$ & $1.5 e-5$ & 0.4 & 0.22 & 0.16 & 0.39 \\
\hline
\end{tabular}

model. The values of the fitting parameters under a leastsquare criterium are listed in Table 1.

See that expression (1) models the magnitude of the frequency response. This is because for calculating the capacity of a channel only the magnitude of the frequency response is relevant and no attention needs to be paid to the phase response, as indicated by the coming expression (7) in the next section.

\section{Frequency Response Extrapolation}

Once the frequency response of the channel $H_{r_{0}}(f)$ is known for a certain distance $r_{0}$ between transmitter and receiver, our goal is to extrapolate this result and obtain the frequency response $H_{r}(f)$ at different arbitrary distance $r$. To do so, a propagation model must be first recalled.

In a homogeneous conducting medium, the main component of the electric field radiated by a linear dipole antenna at a certain distance $r$ is given by [7]

$$
E=j \eta \frac{k I_{0} l \sin (\theta)}{4 \pi r}\left(1+\frac{1}{j k r}-\frac{1}{(k r)^{2}}\right) e^{-j k r} \hat{\theta},
$$

where $I_{0}$ and $l$ are the antenna current and length, respectively, and $\theta$ is the polar angle of the observation point assuming that the dipole is aligned with the $z$-axis. The characteristic impedance of the medium $\eta$ is given by

$$
\eta=\sqrt{\frac{\mu}{\epsilon}\left(1-j \frac{\sigma}{\omega \epsilon}\right)^{-1}}
$$

where $\omega$ is the angular frequency, $\epsilon, \mu$, and $\sigma$ are the permittivity, permeability, and conductivity of sea water, respectively, and $k$ is the propagation constant whose expression is

$$
k=\omega \sqrt{\mu \epsilon\left(1-j \frac{\sigma}{\omega \epsilon}\right)} .
$$

The measured channel consists of a path of homogeneous medium between two points separated by a certain distance $r_{0}$. If those two points separate a different distance $r=r_{0}+\Delta r$ then the new channel is the series connection of two channels. The new frequency response can be estimated as

$$
H_{r}(f)=H_{r_{0}}(f) \cdot H_{\Delta r}(f)=H_{r_{0}}(f) \cdot \frac{E_{r_{0}+\Delta r}(f)}{E_{r_{0}}(f)},
$$

where $E_{r}(f)$ is the electric field in (2) at a distance $r$ and frequency $f$. Explicit dependence on distance and frequency of the electric field has been included in the notation for convenience. Subtituting (2) in (5) yields

$$
H_{r}(f)=H_{r_{0}}(f) \cdot \frac{r_{0}\left(1+(1 / j k r)-\left(1 /(k r)^{2}\right)\right)}{r\left(1+\left(1 / j k r_{0}\right)-\left(1 /\left(k r_{0}\right)^{2}\right)\right)} e^{-j k\left(r-r_{0}\right)} .
$$




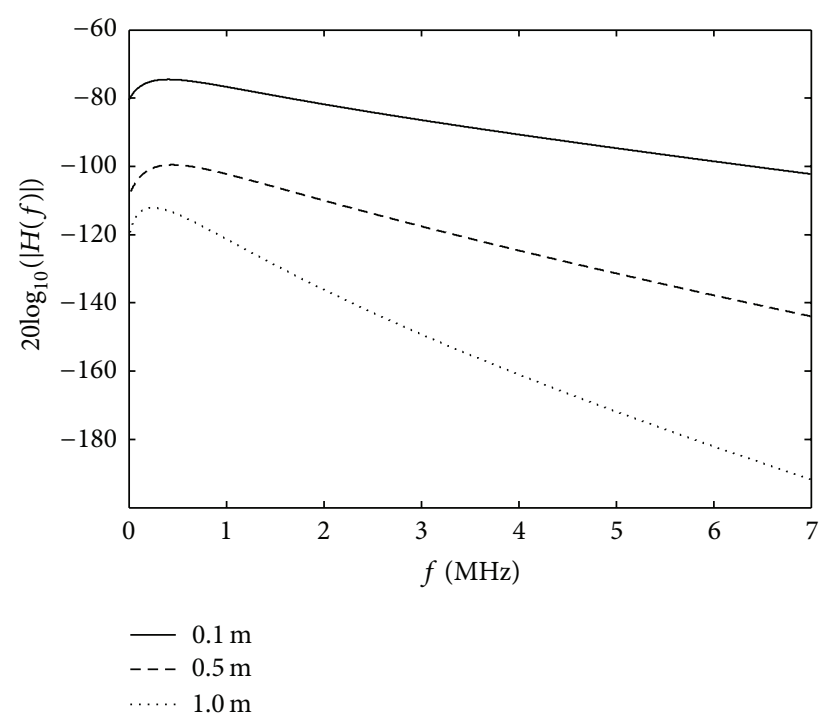

FIGURE 2: Frequency response of the channel for reference distance $r_{0}=0.1 \mathrm{~m}$ and for extrapolated distances $r=0.5 \mathrm{~m}$ and $r=1.0 \mathrm{~m}$.

With expression (6) the channel frequency response can be extrapolated to any distance $r$. Figure 2 depicts the frequency response of the underwater channel with the parameters shown in Table 2 for $r_{0}=0.1 \mathrm{~m}, r=0.5 \mathrm{~m}$, and $r=1.0 \mathrm{~m}$.

As expected, the channel magnitude shows a strong attenuation varying from $75 \mathrm{~dB}$ to $100 \mathrm{~dB}$ in the given frequency range for the shortest distance. This attenuation increases significantly with the separation between transmitter and receiver. This increase is more evident in the higher end of the frequency band.

\section{Channel Capacity}

Once the frequency response is available, the capacity of the channel $C_{r}$ for a given separation between transmitter and receiver $r$ can be easily determined assuming the noise is additive white and gaussian (AWG) and using the wellknown Shannon's expression

$$
C_{r}=\int_{0}^{B} \log _{2}\left(1+\frac{S_{x}(f)\left|H_{r}(f)\right|^{2}}{N_{0}}\right) d f,
$$

where $S_{x}(f)$ and $N_{0}$ are the power spectrum density of the transmitted signal and the noise, respectively, and $B$ is the channel bandwidth. Given a total available power $P_{x}$ constraint, the water pouring algorithm can be used to find the $S_{x}(f)$ that maximizes (7) with

$$
P_{x}=\int_{-\infty}^{\infty} S_{x}(f) d f
$$

An important issue must however be considered before solving (7). In the communication system under study the transmitter is assumed to be attached to a subsea infrastructure while the receiver is on board of a ROV. This means that
TABLE 2: Channel parameters.

\begin{tabular}{llc}
\hline Parameter & Description & Value \\
\hline$\sigma$ & Sea water conductivity & $4.3 \mathrm{~S} / \mathrm{m}$ \\
$\epsilon$ & Sea water relative permittivity & 85 \\
$\mu$ & Sea water permeability & $4 \pi \cdot 10^{-7} \mathrm{H} / \mathrm{m}$ \\
$N_{0}$ & Noise power spectral density & $-177 \mathrm{dBm} / \mathrm{Hz}$ \\
\hline
\end{tabular}

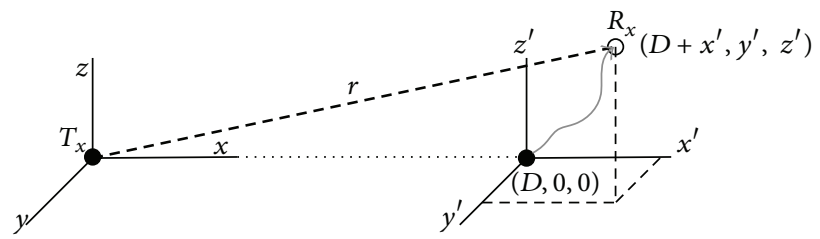

FIGURE 3: Coordinate system for transmitter and receiver location.

the length of the link is not constant over the transmission period but randomly fluctuates due to the water movement. Suppose that the nominal distance between transmitter and receiver is $D$ meters. According to Figure 3, the transmitter is at coordinates $(0,0,0)$ and the receiver is at $(D, 0,0)$. The fluctuations can be accounted for by introducing three normal i.i.d. zero-mean normal random variables $x^{\prime}, y^{\prime}$, and $z^{\prime}$ with standard deviation $\sigma_{x y z}$ so that the new position of the receiver is now at coordinates $\left(D+x^{\prime}, y^{\prime}, z^{\prime}\right)$. The distance from transmitter to receiver changes therefore from a constant $D$ to a random variable $r$ given by

$$
r=\sqrt{\left(D+x^{\prime}\right)^{2}+y^{\prime 2}+z^{\prime 2}} .
$$

Moreover, the random fluctuation of the receiver will also cause a random misalignment of the transmitter and receiver dipole antennas lowering the frequency response gain and the capacity. The misalignment angle can be modeled by a normal random variable $\psi$ with standard deviation $\sigma_{\psi}$. This introduces a factor $\cos (\psi)$ in the frequency response of the channel in (5) and (6).

In this new scenario a measure of the channel capacity is given by the ergodic capacity $C_{\text {erg }}$ defined in [8] as the average of the instantaneous capacity given in (7). Assuming independence between $r$ and $\psi$ the expression for $C_{\text {erg }}$ is

$$
C_{\text {erg }}=E\left[C_{r}\right]=\iint_{-\infty}^{\infty} C_{r} p_{R}(r) p_{\psi}(\psi) d \psi d r,
$$

where $p_{R}(r)$ is the probability density function of the distance $r$ defined in (9) and $p_{\psi}(\psi)$ is the probability density function of the misalignment angle $\psi$.

Substituting (7) into (10) yields

$$
C_{\text {erg }}=E\left[\int_{0}^{B} \log _{2}\left(1+\frac{S_{x}(f)\left|H_{r}(f)\right|^{2}}{N_{0}}\right) d f\right] .
$$

The inner integral in (11) is solved numerically and the expectation is solved by simulation. Results have been obtained in two different scenarios regarding the movement of the sea water: calm water and troubled water. In each one, 
TABLE 3: Simulation parameters.

\begin{tabular}{llc}
\hline Parameter & Description & Value \\
\hline$\sigma_{x y z}$ & Standard deviation of & $0.05 \mathrm{~m}$ (calm water) \\
& $x^{\prime}, y^{\prime}$, and $z^{\prime}$ & $0.25 \mathrm{~m}$ (calm water) \\
$\sigma_{\psi}$ & Standard deviation of $\psi$ & $\pi / 20 \mathrm{rad}$ (calm water) \\
& & $\pi / 5 \mathrm{rad}$ (troubled water) \\
$B$ & Bandwidth & $7 \mathrm{MHz}$ \\
$P_{x}$ & Total available power & $1 \mathrm{~mW}, 5 \mathrm{~mW}$, and $10 \mathrm{~mW}$ \\
\hline
\end{tabular}

the values of both $\sigma_{x y z}$ and $\sigma_{\psi}$ are different. Low values will correspond to calm water and high values to troubled water. The values of the parameters used in the simulation are listed in Table 3. Results are shown in Figure 4 where the ergodic capacity as a function of the distance is plotted for three different values of transmitter power $P_{x}$ and for two sets of values of $\sigma_{x y z}$ and $\sigma_{\psi}$ corresponding to calm and troubled water.

Solid and dashed lines correspond to calm and troubled water, respectively. As can be seen there is a general reduction in the capacity when going from calm to troubled water. The relative reduction is quite uniform over the length of the path. In average terms a drop of around $20 \%$ is experimented in longer distances $(>3 \mathrm{~m})$ and $15 \%$ for shorter distances. This is true for the three values of $P_{x}$.

Regarding the behavior of the capacity with distance, a strong dependence is observed. Values range from tens of $\mathrm{kbps}$ at distances between $5 \mathrm{~m}$ and $3 \mathrm{~m}$, growing to hundreds of kpbs between $3 \mathrm{~m}$ and $1.5 \mathrm{~m}$, and reaching several Mbps in shorter distances. See that capacity values in the order of $10 \mathrm{Mbps}$ and higher are obtained for distances of $0.5 \mathrm{~m}$ and shorter. According to Figure 4, at distances less than $\approx 3 \mathrm{~m}$ the capacity exceeds the maximum data rates achieved by conventional acoustic links ( $40 \mathrm{kbps})$. It is in this shortrange scenario where the electromagnetic alternative finds its new potential, as stated earlier in this paper. Finally, notice that moderate available power $P_{x}$ values have been used in the calculations. An increase of these values (as suggested by some commercial units now on the market [5]) would remarkably improve the results.

\section{Conclusion and Future Work}

The electromagnetic underwater channel capacity has been estimated for the case of very short-range $(<5 \mathrm{~m})$, deep sea communication. A known model for the frequency response has been extrapolated to any channel range and a statistical model has been introduced for the channel to account for the random fluctuation of the receiver location and antenna misalignment. Under these circumstances the ergodic capacity has been calculated with a realistic set of parameters. Results show capacity values ranging from tens of kbps to several Mbps for distances from $5 \mathrm{~m}$ to $0.5 \mathrm{~m}$. Special attention must be paid to the shorter distances $(<1 \mathrm{~m})$ where most of the new applications are developing. Capacity values up to $10 \mathrm{Mbps}$ set a new horizon for future underwater electromagnetic communication systems.

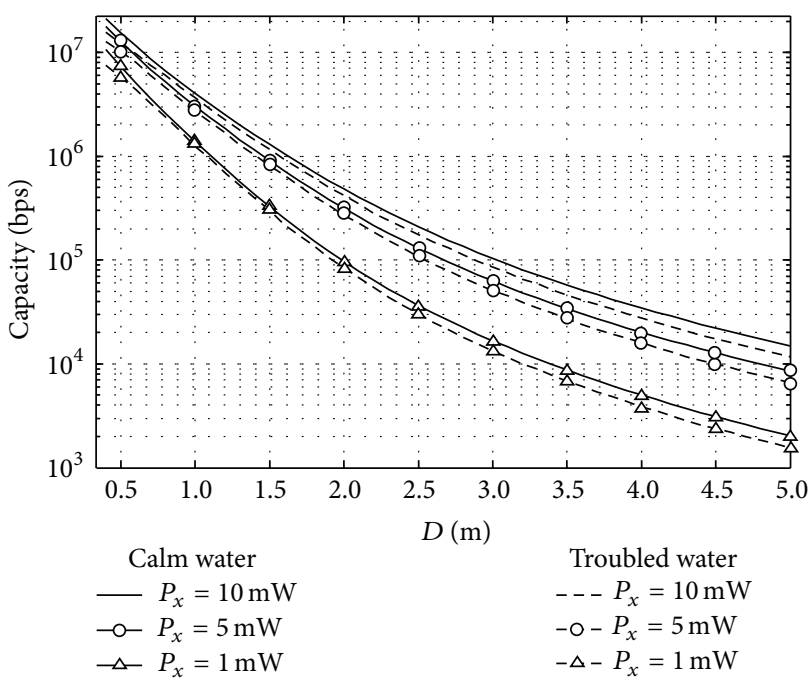

FIGURE 4: Ergodic capacity as a function of distance $D$ for different values of $P_{x}$ and for two scenarios: calm and troubled water.

The authors are working towards the experimental validation of the results. A measurement equipment is being set up to be eventually loaded on a small boat. Frequency response measurements at separations other than $0.1 \mathrm{~m}$ between transmitter and receiver will be made to validate the extrapolation model presented in this work.

\section{Conflict of Interests}

The authors declare that there is no conflict of interests regarding the publication of this paper.

\section{Acknowledgments}

This work has been partially supported by FEDER and the Spanish and Andalusian Governments, under projects TEC2011-25473 and P11-TIC-8238, respectively. This work has also been partially supported by the project SUMERGI + DOS which is funded by the Centro para el Desarrollo Tecnológico Industrial (CDTI) and cofunded by FEDER through the FEDER-INNTERCONECTA research program.

\section{References}

[1] J. Joe and S. H. Toh, "Digital underwater communication using electric current method," in OCEANS 2007-Europe, pp. 1-4, June 2007.

[2] X. Che, I. Wells, G. Dickers, P. Kear, and X. Gong, "Reevaluation of RF electromagnetic communication in underwater sensor networks," IEEE Communications Magazine, vol. 48, no. 12, pp. 143-151, 2010.

[3] J. Li and M. Toulgoat, "EM channel characteristics and their impact on MAC layer performance in underwater surveillance networks," Wireless Communications and Mobile Computing, 2014.

[4] Z. Wu, J. Xu, and B. Li, "A high-speed digital underwater communication solution using electric current method," in 
Proceedings of the 2nd International Conference on Future Computer and Communication (ICFCC '10), pp. V2-14-V2-16, Wuhan, China, May 2010.

[5] Wireless For Subsea (WFS), Subsea Wireless Instrumentation and Control Solutions, 2014, http://www.wfs-tech.com/.

[6] A. Zoksimovski, D. Sexton, M. Stojanovic, and C. Rappaport, "Underwater electromagnetic communications using conduction-channel characterization," in Proceedings of the 7th ACM International Conference on Underwater Networks and Systems (WUWNet '12), Los Angeles, Calif, USA, November 2012.

[7] C. A. Balanis, Antenna Theory, Analysis and Design, chapter 4, John Wiley \& Sons, New York, NY, USA, 1999.

[8] A. Goldsmith, Wireless Communications, chapter 4, Cambridge University Press, 2005. 

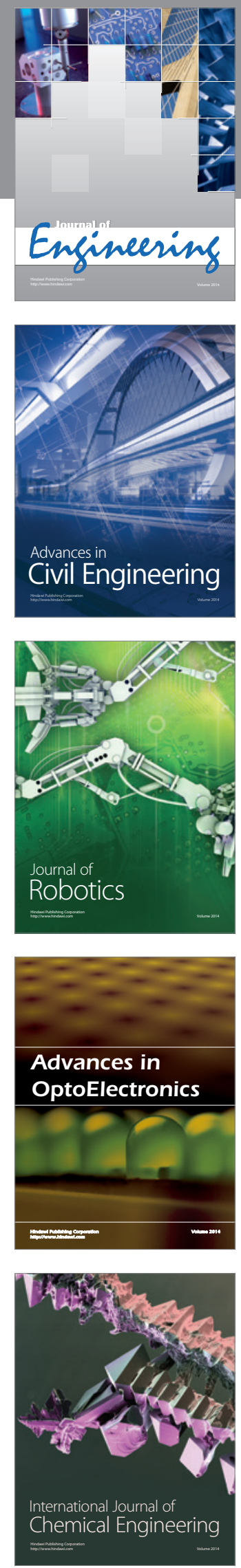

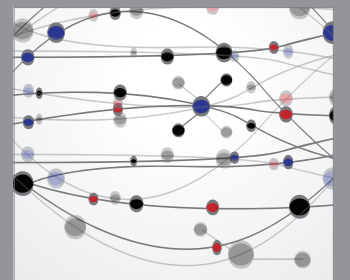

The Scientific World Journal
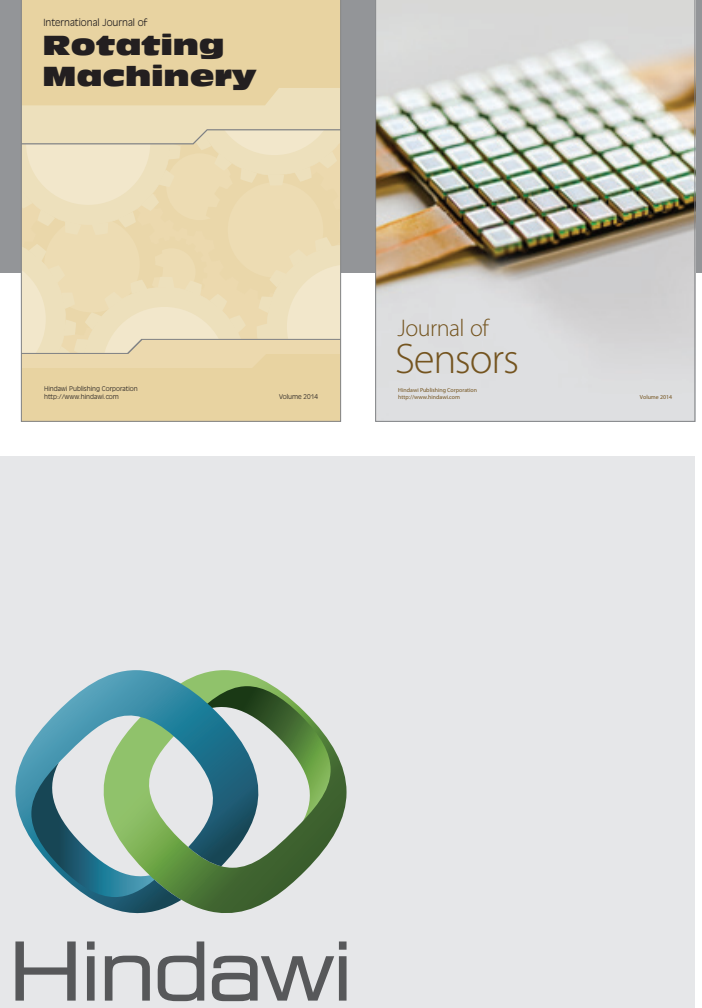

Submit your manuscripts at http://www.hindawi.com
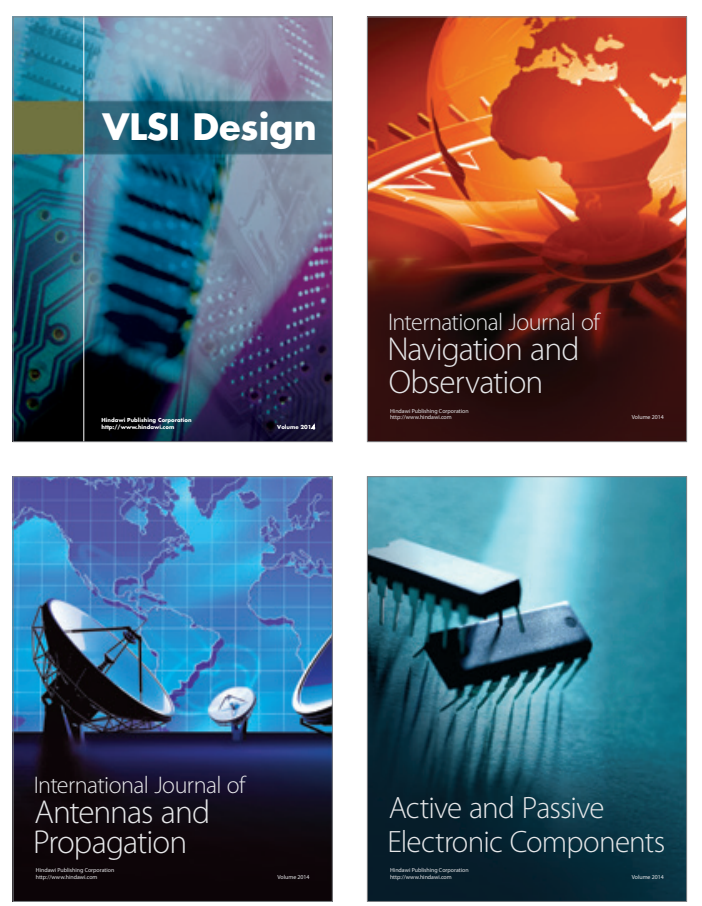
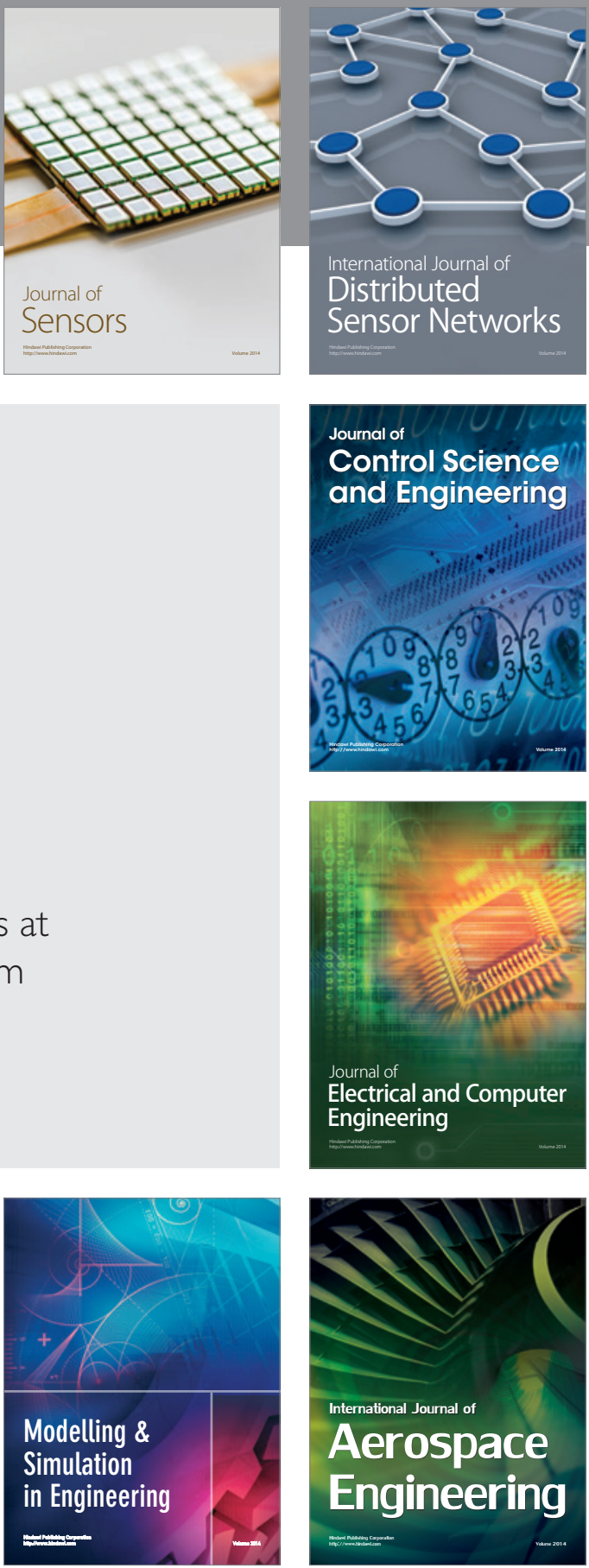

Journal of

Control Science

and Engineering
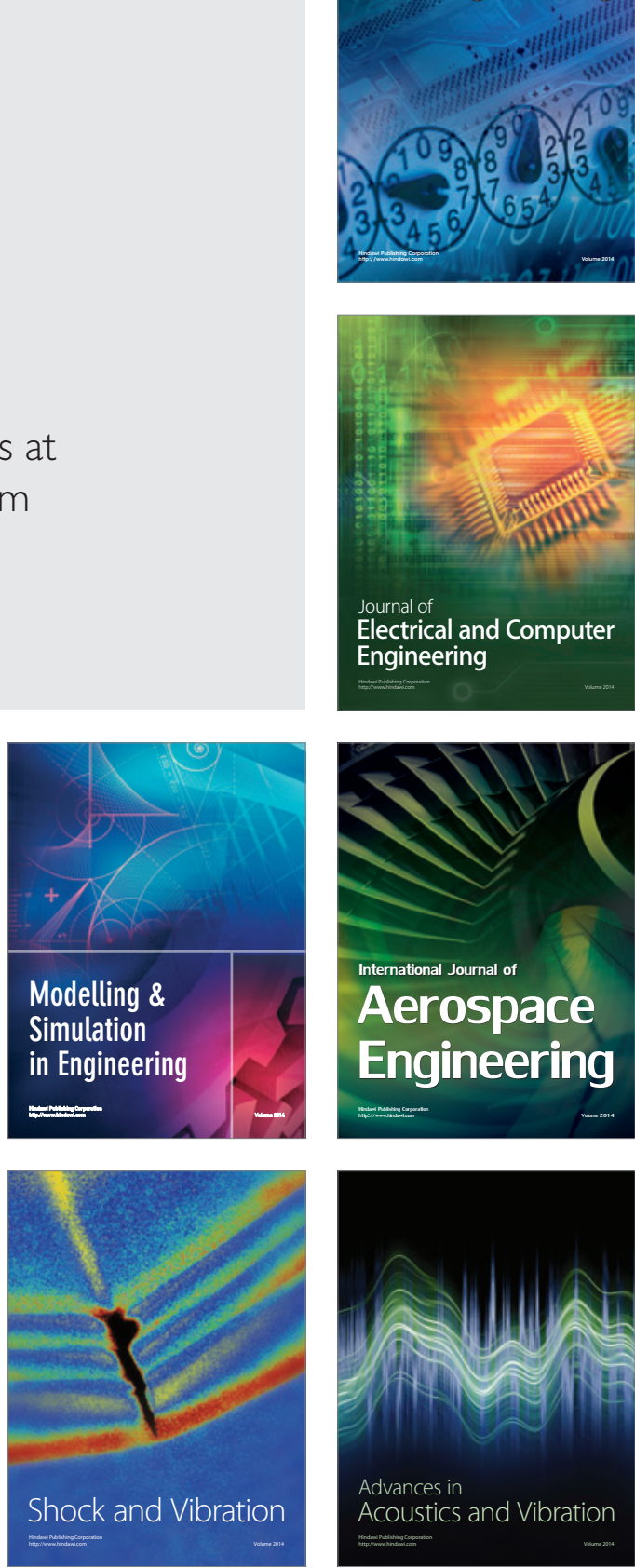